\title{
Efecto de la recirculación de polvo de fundición de cobre de Chile con altos contenidos de impurezas en la distribución de impurezas durante el proceso de fusión ${ }^{(\cdot)}$
}

\author{
V. Montenegro*, H. Sano** y T. Fujisawa**
}

Resumen

\begin{abstract}
Usualmente, el polvo generado durante el proceso de fusión de concentrado de cobre por el Convertidor Teniente y el Horno Flash en Chile, contienen altos contenidos de cobre, zinc, arsénico y antimonio, entre otros metales. En general, el polvo se recircula al proceso de fusión o se envía a procesos hidrometalúrgicos para recuperación y estabilización. Sin embargo, en los años recientes, la generación de polvo ha aumentado debido a la baja en la calidad del concentrado. Adicionalmente, las normas ambientales se han vuelto más estrictas. Por lo tanto, es deseable entender el comportamiento de estos elementos cuando el proceso opera con recirculación de polvos. En este estudio, el efecto de la recirculación de polvo al proceso de fusión de cobre en la distribución entre la mata, escoria y gas fue evaluado como una función del grado de la mata, cantidad de polvo recirculado, enriquecimiento de oxígeno y temperatura. Se encontró que la concentración en el eje, de metales tales como, arsénico, antimonio y bismuto aumentaron levemente con el polvo recirculado. Por otro lado, la concentración de plomo y zinc depende fuertemente de la recirculación directa de polvo al proceso. Adicionalmente, se encontró que altos niveles de arsénico y antimonio en el polvo, pueden provocar formación y precipitación de arseniatos de cobre y otros metales (speiss), los cuales pueden provocar problemas operacionales de importancia.
\end{abstract}

\section{Recirculation effect of Chilean copper smelting dust with high impurities contents on the impurity distributions during smelting process}

\begin{abstract}
Usually, dust generated during the copper smelting process by the Teniente Converter and the Flash Smelting Furnaces in Chile, contains high concentrations of copper, zinc, arsenic, antimony and other metals. In general, the dust is recirculated to the smelting process or it is directed to hydrometallurgical process for recovery and stabilization. However, in recent years the generation of dust has increased because of the degradation of the quality of the concentrate. In addition, the environmental regulations have become stricter. It is therefore desirable to understand the behavior of those elements, when the smelting process operates with recirculation of dust. In this study, the effect of dust recirculation to smelting process on the distribution among the matte, slag and gas phases was evaluated, as a function of matte grade, amount of recirculated dust, oxygen enrichment and temperature. It was found that the concentration in the matte of the impurities such as arsenic, antimony and bismuth, increased slightly with recirculation of dust. On the other hand, the concentration of lead and zinc depend of the direct recirculation of dust to the process. Additionally, it was found that high concentrations of arsenic and antimony in the dust may lead to the formation and precipitation of copper arsenates and other metals (speiss), which may generates important operational problems.
\end{abstract}

Keywords Minor elements distribution; Dust recirculation; Copper smelting dust.

\section{INTRODUCCIÓN}

La remoción de impurezas en el proceso de fusión de cobre se produce mediante oxidación seguido por escorificación o volatilización durante las etapas de fusión, conversión y refino ${ }^{[1}$ y 2]. Los polvos generados durante el proceso de producción de cobre contienen altas concentraciones de $\mathrm{SO}_{2}$, metales pesados y

\footnotetext{
(•) Trabajo recibido el día 2 de abril de 2009 y aceptado en su forma final el día 20 de octubre de 2009.

* Department of Materials Science and Engineering, Graduate School of Engineering, Nagoya University, Nagoya 464-8603, Japan. Email:vmontene@gmail.com

${ }^{* *}$ EcoTopia Science Institute, Nagoya University, Furo-cho, Chikusa-ku, Nagoya 464-8603, Japan.
} 
otras impurezas. Actualmente, las emisiones de polvos representan un problema serio en la metalurgia mundial, especialmente en Chile, donde el concentrado de cobre contiene metales peligrosos para el organismo humano. Aun cuando las emisiones desde las fundiciones de cobre se han reducido en los años recientes, los problemas ambientales de contaminación de suelos aun son de mucha importancia, además las normas ambientales se han vuelto más estrictas, lo cual hace necesario revisar nuevas alternativas de recuperación de metales y tratamiento de polvos metalúrgicos.

El polvo generado durante el proceso de fusión es dependiente del tipo de reactor. El convertidor Teniente genera alrededor del $1 \%$ de su carga como polvo y el proceso Flash alrededor de $4-10 \%{ }^{[3]}$. Las grandes cantidades de polvo generado requieren ser reducidas y recuperar los elementos de interés económico que están contenidos. Usualmente, las mayores impurezas en el polvo son arsénico (As), plomo $(\mathrm{Pb})$, zinc $(\mathrm{Zn})$, bismuto $(\mathrm{Bi})$ y antimonio $(\mathrm{Sb})$, además de con un contenido importante de cobre. As, $\mathrm{Pb}, \mathrm{Bi}$ y $\mathrm{Zn}$ son metales peligrosos con un riesgo potencial de movilización y transferencia hacia otros componentes del ambiente, tales como plantas y aguas subterráneas. Zn genera problemas operacionales ya que juega un importante rol en la creación de acreciones en hornos y trenes de gases, especialmente en el proceso de fusión flash. Adicionalmente, las impurezas presentes en el proceso, tales como As y $\mathrm{Sb}$, requieren un control especial en la refinería electrolítica para mantener los estándares de calidad ${ }^{[4-5]}$.

En este estudio, se evalúo el efecto de la recirculación directa de polvo en el proceso con varias adiciones de polvo al proceso, lo cual permite determinar la distribución de los elementos entre los productos como una función del polvo recirculado, grado del eje, enriquecimiento de oxigeno y la temperatura. Las distribuciones de los elementos menores permiten determinar su efecto en la calidad de los productos y a los procesos posteriores de conversión y refinación de cobre.

\section{MÉTODO EXPERIMENTAL}

Muestras industriales de concentrado de cobre, eje, escoria y polvos provenientes de Convertidor Teniente, de una fundición de cobre de Chile, fueron utilizados en este estudio. La tabla I muestra la composición química de las muestras. Los resultados de los análisis químicos y XRD de los polvos muestran que los principales componentes son $\mathrm{CuSO}_{4}, \mathrm{Cu}_{2} \mathrm{O}$, $\mathrm{PbSO}_{4}, \mathrm{ZnSO}_{4}, \mathrm{ZnO}_{\text {y As}} \mathrm{O}_{3}$ (Fig. 1).

Los experimentos fueron diseñados y desarrollados en un horno eléctrico, escala laboratorio, como se presenta esquemáticamente en la figura 2 . Inicialmente, se cargaron $13 \mathrm{~g}$ de escoria, $10 \mathrm{~g}$ eje de cobre y $20 \mathrm{~g}$ de carga (concentrado, polvo y sílice mezclados en diferentes razones) en un crisol de alúmina y fueron posicionados en un tubo de reacción $(12 \mathrm{~mm}$ I.D., $100 \mathrm{~mm} \mathrm{H}$.) sellado con flanches de bronce enfriados por agua. El horno fue calentado hasta una temperatura previamente determinada (entre 1.523. $1.573 \mathrm{~K}$ ), bajo un flujo continuo de nitrógeno de 200 $\mathrm{cm}^{3} \cdot \mathrm{min}^{-1}$, para mantener una atmosfera neutra. Un termopar $\mathrm{Pt} / \mathrm{Pt}$-Rh cubierto con una vaina de alúmina se introdujo en el horno para monitorear la temperatura. Después de la fusión y estabilización de la temperatura, se introdujo un tubo de alúmina para iniciar la inyección de gas.

Los gases provenientes del tubo de reacción se pasaron continuamente por un condensador enfriado por agua para recolectar los elementos volátiles y, posteriormente, se pasaron por soluciones de $1 \mathrm{M} \mathrm{H}_{2} \mathrm{SO}_{4}$ y $1 \mathrm{M} \mathrm{NaOH}$ para remover los elementos peligrosos y neutralizar el $\mathrm{SO}_{2}$. Cuando la inyección de gas de soplado finalizó, la muestra fue mantenida a la misma temperatura por $30 \mathrm{~min}$ para promover la sedimentación y separación de fases. Luego, el crisol fue removido desde el horno y enfriado inmediatamente

Tabla I. Composición química de las muestras

Table I Chemical composition of samples.

\begin{tabular}{lrrrrrrrr}
\hline \multicolumn{1}{c}{ Elementos } & Cu & \multicolumn{1}{c}{ Fe } & S & Zn & Pb & Bi & As & Sb \\
\hline Concentrado & 36,1 & 22,9 & 32,6 & 0,7 & 0,2 & 0,1 & 0,7 & 0,01 \\
Mata & 76,4 & 1,4 & 19,8 & 0,6 & 0,1 & ND & 0,4 & 0,01 \\
Escoria & 8,0 & 38,4 & 1,9 & 7,9 & 0,1 & 0,1 & 0,4 & 0,06 \\
Polvo & 10,4 & 0,8 & 10,4 & 15,6 & 7,8 & 3,5 & 19,4 & 0,10 \\
\hline
\end{tabular}




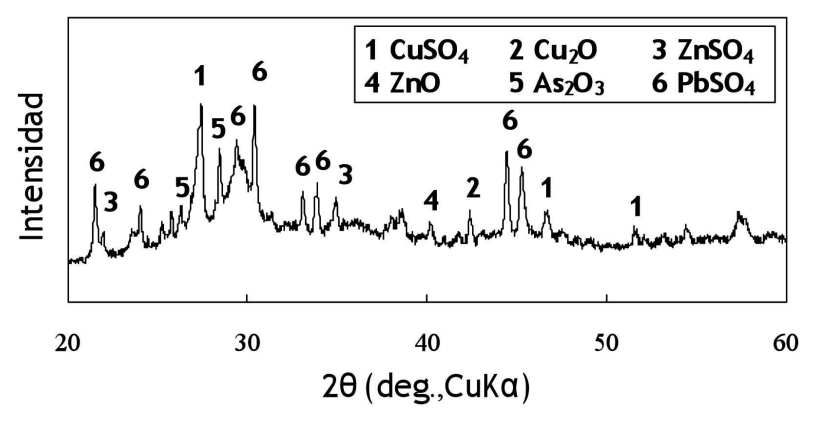

Figura 1. Resultados XRD para polvos de fundición de cobre de Chile.

Figure 1. XRD results of Chilean Smelter Copper Dust.

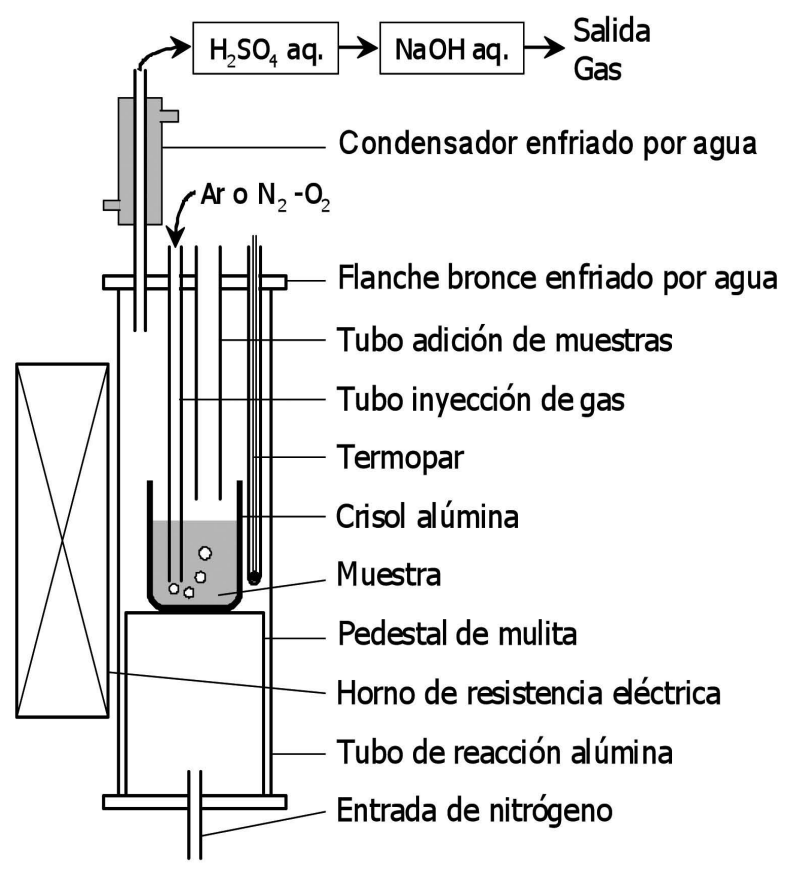

Figura 2. Aparatos experimentales.

Figure 2. Experimental apparatus.

usando un flujo de gas helio. El material condensado, fases de mata y escoria, se analizaron por su composición elemental, usando ICP-AES (inductively coupled plasma atomic emission spectrometry) y difracción de rayos $\mathrm{X}(\mathrm{XRD})$ para determinar las especies presentes en las fases.

Para tratar de reproducir los resultados industriales se utilizaron las mismas razones de masa de eje, escoria y carga que utiliza el Convertidor Teniente Industrial en fundiciones Chilenas ${ }^{[6]}$. La adición de fundente fue ajustada para mantener una razón en escoria de $\mathrm{Fe} / \mathrm{SiO}_{2}$ igual a 2.

\section{RESULTADOS Y DISCUSIONES}

\subsection{Cantidad de polvo recirculado}

La razón de recirculación representa la cantidad de polvo recirculado al proceso, la cual se define por la siguiente expresión:

$$
\text { Razón Recirculación }(\%)=\frac{\text { Cantidad de polvo recirculado }}{\text { Carga (ConcentradrotPolvo+Fundente) }} \times 100
$$

Las distribuciones de $\mathrm{As}, \mathrm{Sb}, \mathrm{Bi}, \mathrm{Pb}$ y $\mathrm{Zn}$ fueron examinadas variando la cantidad de recirculación de polvo al proceso de fusión, utilizando gas de inyección con $21 \%$ de oxigeno a $1.523 \mathrm{~K}$. La figura 3 presenta los resultados obtenidos. Los términos $<\% \mathrm{X}>$, $(\% \mathrm{X})$ y $\{\% \mathrm{X}\}$ representan la distribución fraccionada del elemento entre las fases de gas, escoria y eje, respectivamente. Los puntos sólidos representan la concentración del elemento en el eje. Como se observa en la figura, la concentración de As, Bi y Zn aumenta drásticamente en el eje cuando aumenta la recirculación de polvo. La volatilización de Bi es casi independiente de la recirculación de polvos. Por otro lado, las concentraciones de $\mathrm{Sb}$ y $\mathrm{Pb}$ aumentan levemente. La cantidad de $<\% \mathrm{As}>\mathrm{y}<\% \mathrm{Bi}>$ aumentaron, pero $<\% \mathrm{Sb}>,<\% \mathrm{~Pb}>$ y $<\% \mathrm{Zn}>$ disminuyeron. Como disminuyó la volatilización de $\mathrm{Zn}$ y $\mathrm{Pb}$, aumento la eliminación vía escorificación, lo cual se atribuye a que el $\mathrm{Pb}$ y $\mathrm{Zn}$, originalmente, se encuentran en forma de óxidos en el polvo, como se presento anteriormente en la figura 1.

Itagaki y Yazawa ${ }^{[7}$ y 8$]$ demostraron que a $1.573 \mathrm{~K}$, la distribución de $\mathrm{As}, \mathrm{Sb}$ y $\mathrm{Bi}$, en la fase eje, aumenta cuando aumenta su concentración en la carga, especialmente para As, que es fuertemente dependiente de la concentración inicial, y la volatilización de $\mathrm{Sb}$ y $\mathrm{Bi}$ son casi independientes de la concentración inicial de la carga. Los resultados de la presente investigación concuerdan con dichas evaluaciones.

La figura 4 muestra un ejemplo de los análisis de difracción de rayos $\mathrm{X}$ de escoria y eje. Las impurezas se encuentran como elementos menores y es muy difícil distinguir las especies presentes, debido a un efecto de apantallamiento con las especies de mayor concentración en las muestras.

\subsection{Grado de eje}

El grado de eje (concentración de cobre en eje) es uno de los parámetros de operación mas importantes del proceso de fusión. La distribución de elementos menores entre las diferentes fases se examinó 

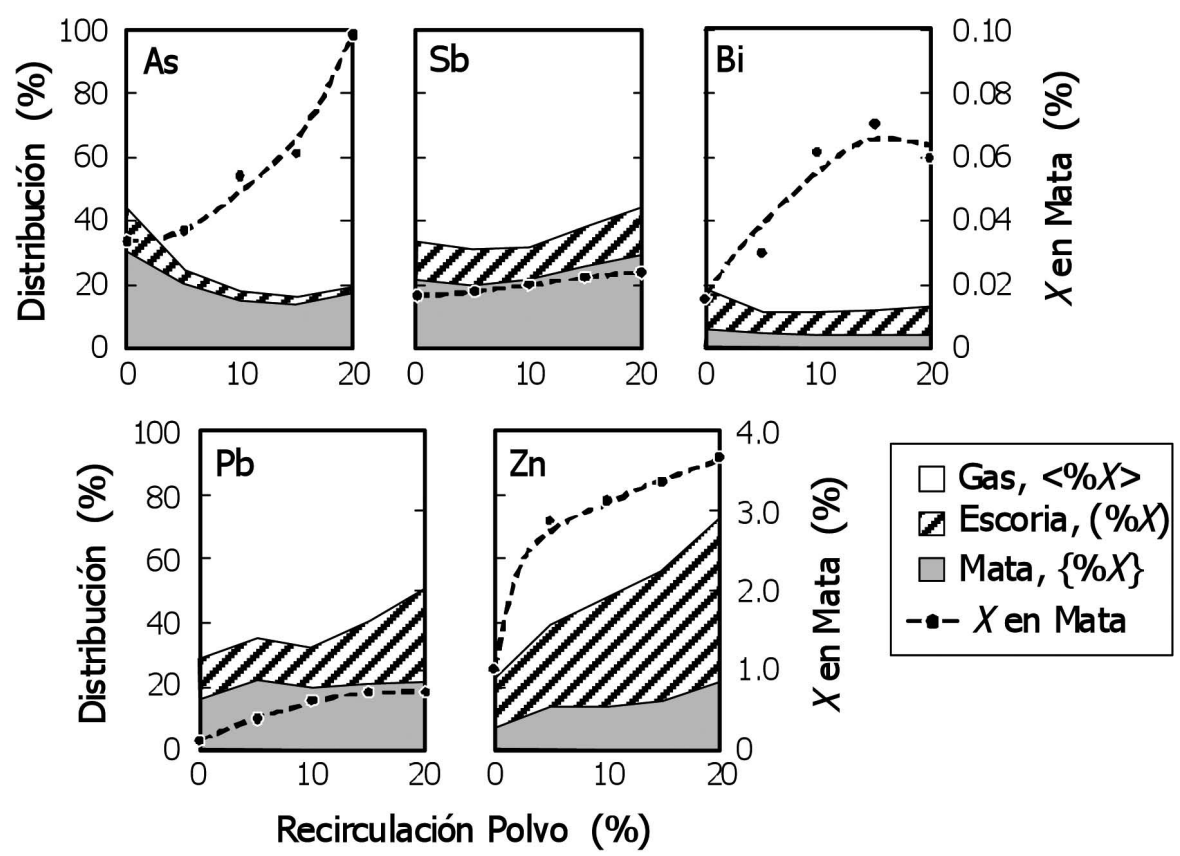

Figura 3. Distribución de $\mathrm{As}, \mathrm{Sb}, \mathrm{Bi}, \mathrm{Pb}$ y $\mathrm{Zn}$ en mata, escoria y gas en relación a la razón de recirculación de polvo a $1.523 \mathrm{~K}$, grado de eje $60 \% \mathrm{Cu}$.

Figure 3. Distribution of $\mathrm{As}, \mathrm{Sb}, \mathrm{Bi}, \mathrm{Pb}$ and $\mathrm{Zn}$ among matte, slag and gas phases in relation to dust recirculation ratio at $1,523 \mathrm{~K}$, matte grade $60 \% \mathrm{Cu}$.
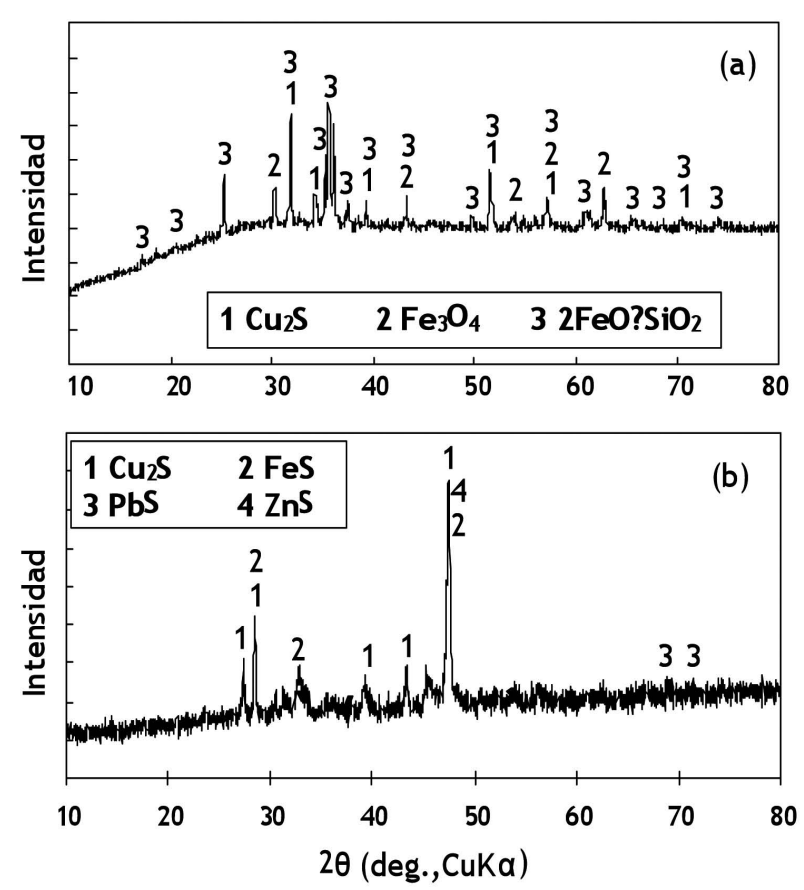

Figura 4. Resultados XRD para (a) escoria y (b) eje para muestras con recirculación de polvos, $5 \%$ de recirculación de polvos a $1.523 \mathrm{~K}$.

Figure 4. XRD results for (a) slag and (b)matte after dust recirculation test, $5 \%$ dust recirculation at $1,523 \mathrm{~K}$. variando la concentración de cobre a $1.523 \mathrm{~K}$, para diferentes razones de recirculación. Se encontró que la distribución de elementos menores en la fase gaseosa aumentó levemente y disminuyó en el eje con el aumento del grado del eje. La distribución en la fase escoria aumentó levemente, ya que aumenta el grado de oxidación de los elementos. En general, los resultados presentaron la misma tendencia para todas las razones de recirculación examinadas. Un ejemplo de los resultados obtenidos con $5 \%$ de recirculación se presenta en la figura 5.

La razón de distribución de varios elementos durante el proceso de fusión de cobre, ha sido intensamente investigada por diferentes autores ${ }^{[9-11]} \mathrm{La} \mathrm{fi-}$ gura 6 presenta el efecto del grado del eje en la razón de distribución entre escoria y eje para $\mathrm{As}, \mathrm{Sb}$, $\mathrm{Pb}$ y $\mathrm{Cu}$, estudiado por Yazawa et al. ${ }^{[11]}$, junto con los resultados del presente trabajo. Los resultados actuales se obtuvieron a $1.523 \mathrm{~K}$, sin precipitación de cobre. La presión parcial de $\mathrm{SO}_{2}$ en el sistema se estimó que fue de alrededor de 0,2 atm, asumiendo que todo el oxígeno alimentado fue convertido en $\mathrm{SO}_{2}$. Aun cuando Yazawa y sus colaboradores desarrollaron los experimentos a $1.573 \mathrm{~K}$, con una presión $\mathrm{PSO}_{2}=0,1$ $\mathrm{atm}$, tales diferencias no producen un efecto significativo en la distribución de los elementos. Ambos resultados presentan un buen ajuste, por lo que se 

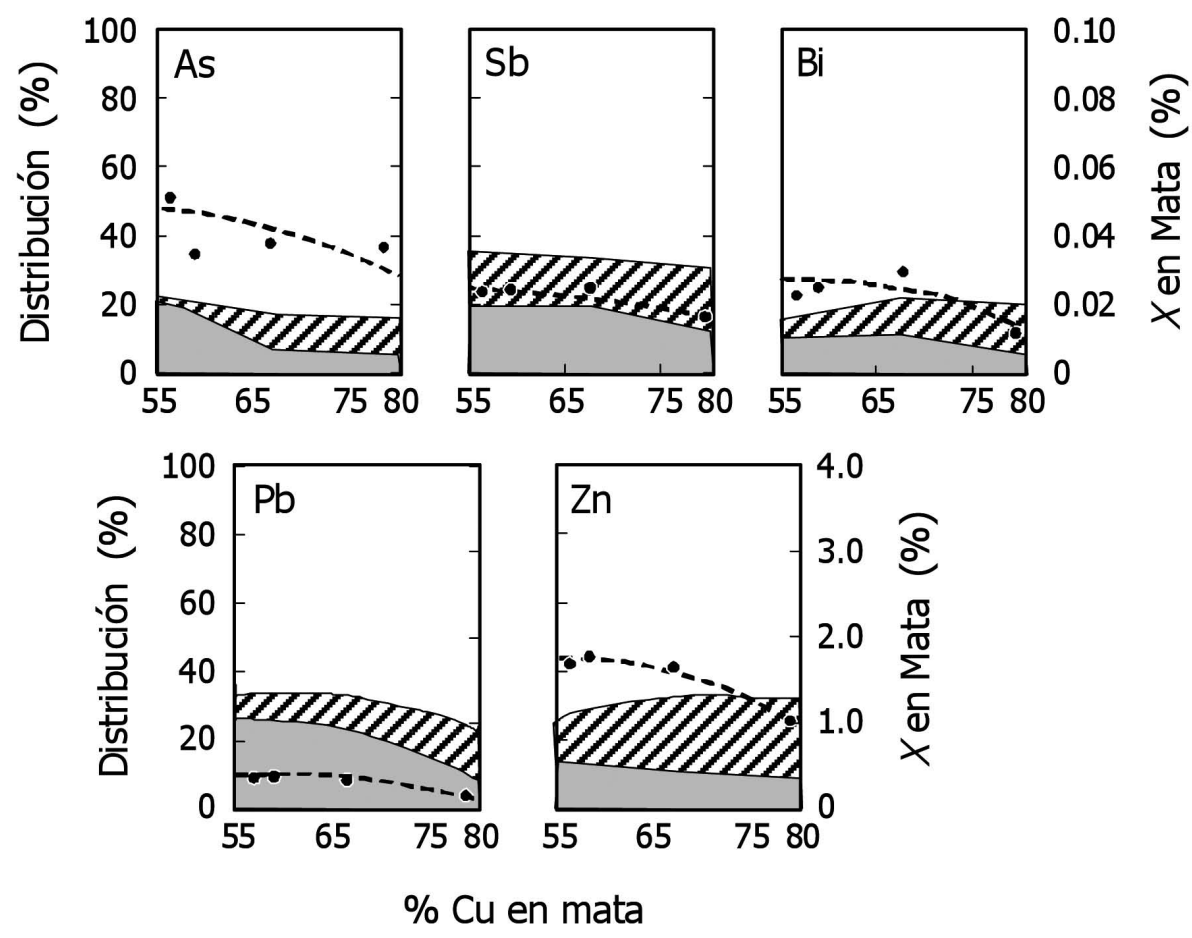

Figura 5. Distribución de $\mathrm{As}, \mathrm{Sb}, \mathrm{Bi}, \mathrm{Pb}$ y $\mathrm{Zn}$ en mata, escoria y gas en relación al grado de eje a $1.523 \mathrm{~K}, 5 \%$ recirculación de polvo.

Figure 5. Distribution of $\mathrm{As}, \mathrm{Sb}, \mathrm{Bi}, \mathrm{Pb}$ and $\mathrm{Zn}$ among matte, slag and gas phases against matte grade at $1,523 \mathrm{~K}, 5 \%$ of dust recirculation.

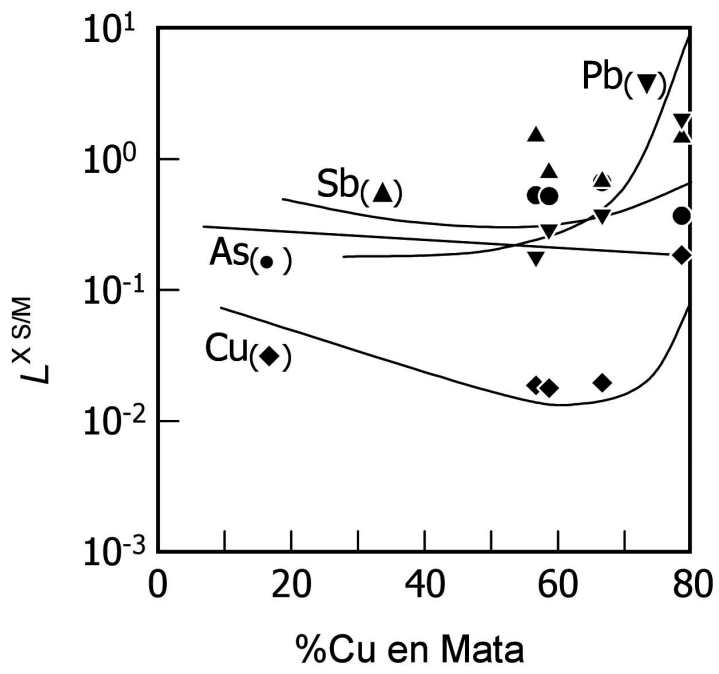

Figura 6. Razón de distribución de As, $\mathrm{Sb}, \mathrm{Pb}$ y Cu entre escoria y eje. (Dibujos: Presente trabajo, $\mathrm{P}_{\mathrm{SO}_{2}} \approx 0,2 \mathrm{~atm}, 1.523 \mathrm{~K}$. Línea sólida: Yazawa ef al., $\left.\mathrm{P}_{\mathrm{SO}_{2}} \approx 0,1 \mathrm{~atm}, 1.573 \mathrm{~K}^{[8]}\right)$.

Figure 6. Distribution ratios of $\mathrm{As}, \mathrm{Sb}, \mathrm{Pb}$ and $\mathrm{Cu}$ between slag and matte. (Plots: Present work, $P_{\mathrm{SO}_{2}} \approx 0.2 \mathrm{~atm}, 1,523 \mathrm{~K}$. Solid line: Yazawa et al., $P_{\mathrm{SO}_{2}} \approx 0.1 \mathrm{~atm}, 1,573 \mathrm{~K}[8]$. estima que el equilibrio entre las fases de escoria y eje se mantuvo durante los experimentos realizados.

\subsection{Enriquecimiento de oxígeno}

La figura 7 presenta el aumento de la concentración de oxígeno en el aire de soplado y su efecto en la distribución de los elementos menores y su contenido en el eje, considerando una razón de recirculación de $5 \%$. Se encontró que la distribución en la fase escoria aumentó con el enriquecimiento de oxígeno. La concentración y distribución de los elementos menores en el eje disminuyó. Esta característica se debe, principalmente, al aumento de la oxidación y escorificación de los elementos con el aumento de la presión parcial de oxígeno.

\subsection{Temperatura}

La figura 8 presenta los resultados del efecto de la temperatura en la distribución de los elementos de interés, con la recirculación de polvo al proceso. La temperatura afecta, principalmente, a la presión de 

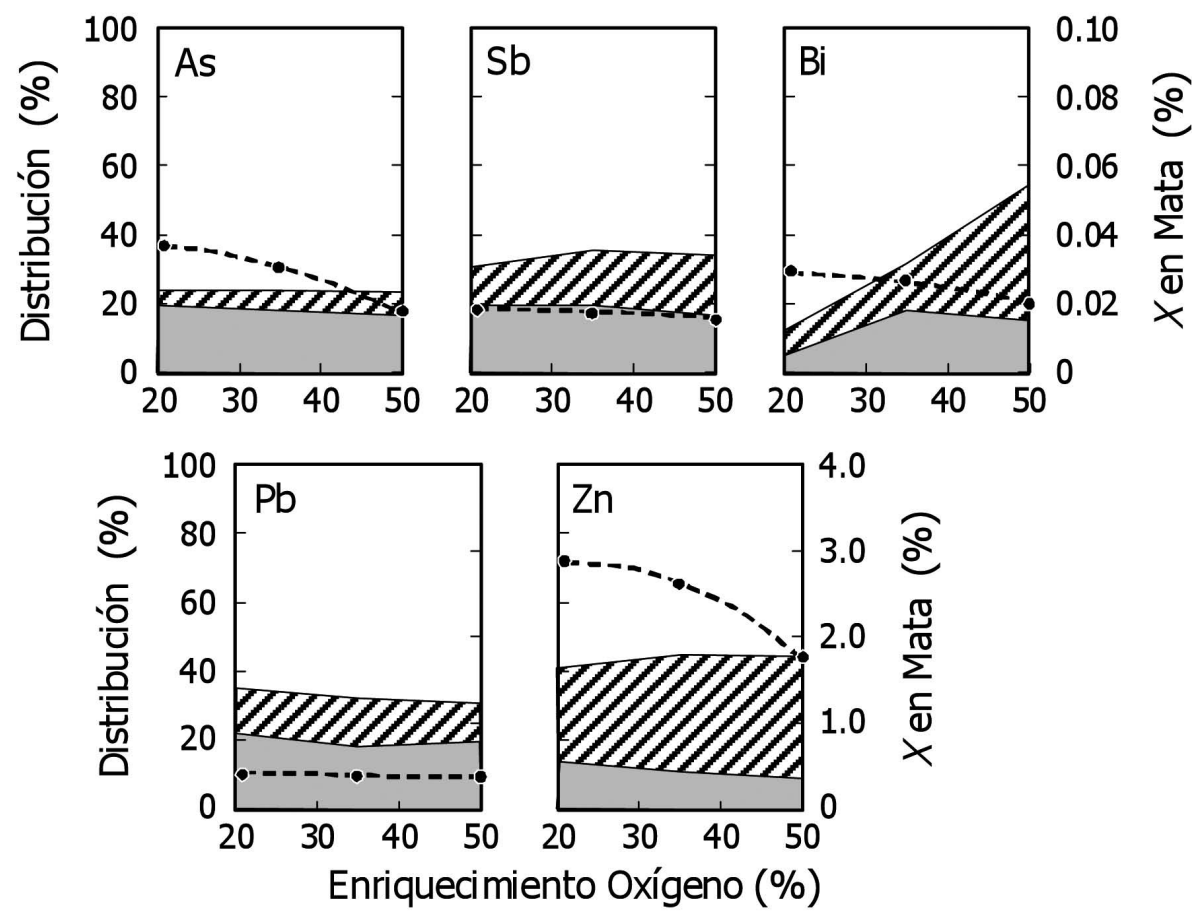

Figura 7. Distribución de As, $\mathrm{Sb}, \mathrm{Bi}, \mathrm{Pb}$ y $\mathrm{Zn}$ en mata, escoria y gas en relación al enriquecimiento de oxigeno en el gas de soplado a $1.523 \mathrm{~K}, 5 \%$ recirculación de polvo

Figure 7. Distribution of $\mathrm{As}, \mathrm{Sb}, \mathrm{Bi}, \mathrm{Pb}$ and $\mathrm{Zn}$ among matte, slag and gas phases against oxygen enrichment in blowing gas at 1,523 K, $5 \%$ dust recirculation.
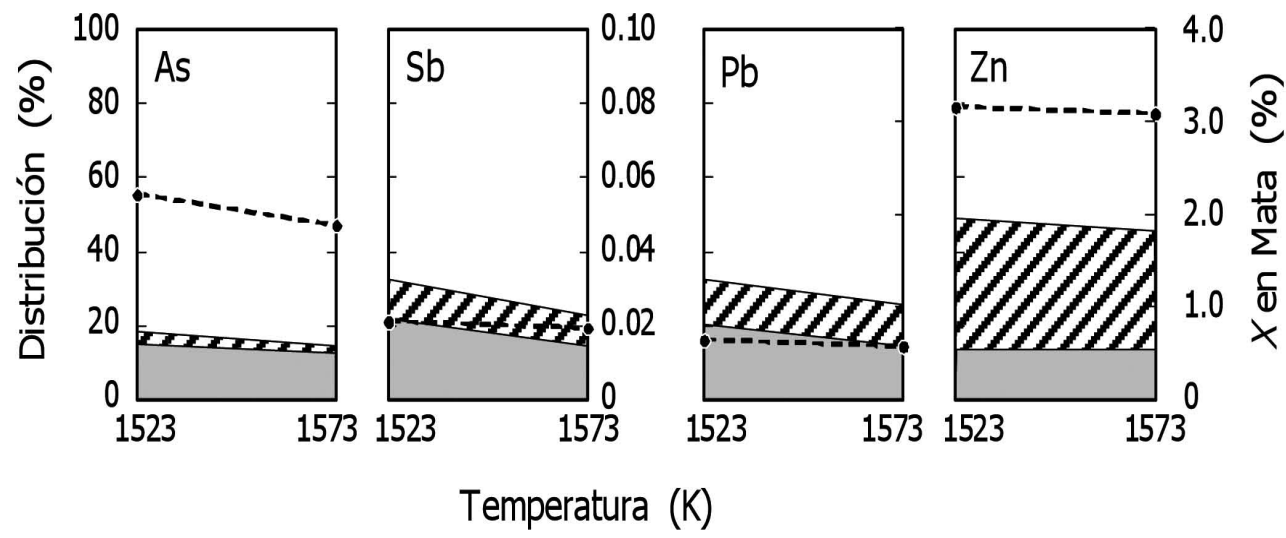

Temperatura $(K)$

Figura 8. Distribución de $\mathrm{As}, \mathrm{Sb}, \mathrm{Bi}, \mathrm{Pb}$ y $\mathrm{Zn}$ en mata, escoria y gas en relación al la temperatura, $10 \%$ recirculación de polvo.

Figure 8. Distribution of $\mathrm{As}, \mathrm{Sb}, \mathrm{Pb}$ and $\mathrm{Zn}$ among matte, slag and gas phases against temperature for the dust recirculation of $10 \%$.

vapor de las especies volátiles de dichos elementos, la cual aumenta con la temperatura. De acuerdo a lo anterior, se espera que el aumento de temperatura permita una mayor eliminación en la fase gaseosa. Los resultados muestran que la concentración de los elementos disminuye lentamente con la temperatura y su efecto es menor en la distribución de los elementos.

Los resultados sugieren que la temperatura no es un parámetro significativo en la distribución de los elementos cuando se recircula polvo al proceso, lo cual es importante desde el punto de vista industrial 
ya que altas temperaturas de operación son difíciles de obtener y, usualmente, dependen de otras propiedades, tales como la viscosidad, consumo de combustible y protección de refractarios, entre otras.

\subsection{Formación de compuestos metálicos de As y Sb}

De acuerdo a los resultados presentados en el presente trabajo, la volatilización de los elementos menores disminuyó con el aumento de la concentración de oxígeno en el gas de inyección, con lo cual disminuyó la generación de polvos y aumentó la eliminación vía escorificación. En cuanto al efecto de la temperatura, se observó que esta no tiene un efecto significativo en la generación de polvos.

La figura 9 muestra el cálculo de la generación de polvos en base a los resultados experimentales, asu-

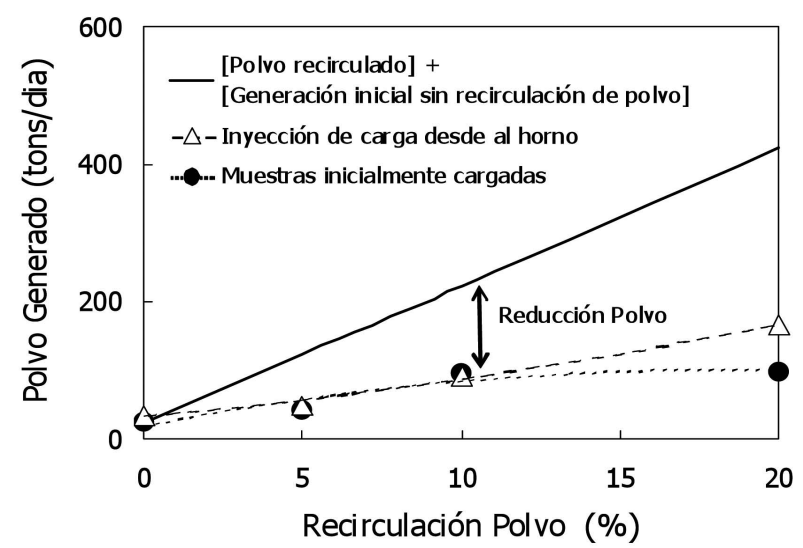

Figura 9. Efecto de la recirculación de polvos en la generación del polvo.

Figure 9. Effect of dust recirculation on the dust generation. miendo un tratamiento de 2.000 ton/d de concentrado de cobre. La línea sólida representa la cantidad de polvos generados, en el caso de asumir que todos los polvos recirculados son eliminados nuevamente por la fase gaseosa. Dichos resultados muestran que la generación de polvos es mucho menor para todos los casos, lo cual se debe a la transferencia de los elementos menores y el cobre a las fases de eje y escoria. La tabla II muestra un ejemplo de la composición química de los productos obtenidos. Para todas las condiciones testeadas, casi no se observaron cambios en el contenido de cobre en la escoria, comparados con la muestra inicial.

Estos resultados demuestran que la recirculación de polvos es efectiva para la recuperación del contenido de cobre de los polvos, pero aumenta la concentración de impurezas en el sistema, creando un problema adicional para los procesos de conversión y refino, ya que las impurezas deben ser eliminadas en las etapas posteriores de modo que se mantenga la calidad del ánodo y del cátodo.

El aumento de la recirculación de polvos tiende a aumentar la concentración de impurezas tales como As y Sb. Cuando el polvo se retorna a la etapa de fusión, el cobre se encuentra presente en forma óxido y sulfato, el arsénico se encuentra presente mayoritariamente como óxido. El aumento de la temperatura por encima de los $900^{\circ} \mathrm{C}$ produce una descomposición térmica del sulfato de cobre, que resulta en la formación de óxido de cobre, de acuerdo a las siguientes reacciones:

$$
\begin{gathered}
\mathrm{CuSO}_{4 \text { polvo }} \Rightarrow(\mathrm{CuO})_{\text {escoria }}+\left\{\mathrm{SO}_{2}\right\}_{\text {gas }}+1 / 2\left\{\mathrm{O}_{2}\right\}_{\text {gas }} \\
3 \mathrm{CuSO}_{4 \text { polvo }} \Rightarrow\left(\mathrm{Cu}_{2} \mathrm{O}\right)_{\text {escoria }}+[\mathrm{Cu}]_{\text {eje }}+3\left\{\mathrm{SO}_{2}\right\}_{\text {gas }}+5 / 2\left\{\mathrm{O}_{2}\right\}_{\text {gas }}
\end{gathered}
$$

Por otra parte, cuando los polvos son recirculados al reactor, éstos no reaccionan como el concentrado sino que forman gotas líquidas en el baño. Los

Tabla II. Composición química de los productos finales en experimentos con $20 \%$ de recirculación de polvos a $1.523 \mathrm{~K}$ con $21 \% \mathrm{O}_{2}$

Table II. Chemical composition of the final products in $20 \%$ of dust recirculation experiment at $1,523 \mathrm{~K}$ with $21 \% \mathrm{O}_{2}$

\begin{tabular}{lcccccccc}
\hline Elementos & $\mathbf{C u}$ & $\mathbf{F e}$ & $\mathbf{S}$ & $\mathbf{Z n}$ & $\mathbf{P b}$ & $\mathbf{B i}$ & $\mathbf{A s}$ & $\mathbf{S b}$ \\
\hline Mata & 61,0 & 13,3 & 23,5 & 3,3 & 0,86 & 0,06 & 0,09 & 0,01 \\
Escoria & 9,5 & 24,2 & ND & 4,3 & 0,47 & 0,03 & 0,03 & 0,02 \\
Polvo & 0,5 & ND & 3,3 & 1,7 & 6,47 & 2,16 & 57,6 & 0,21 \\
\hline
\end{tabular}


óxidos tienden a disolverse en la escoria junto con el $\mathrm{As}_{2} \mathrm{O}_{3}$ y las otras impurezas oxidadas. La presencia de altas cantidades de cobre y arsénico en contacto con el eje promueve la formación de arseniatos, de acuerdo a:

$$
\begin{gathered}
3[\mathrm{FeS}]_{\mathrm{eje}}+7\left(\mathrm{Cu}_{2} \mathrm{O}\right)_{\mathrm{esc}}+\left(\mathrm{As}_{2} \mathrm{O}_{3}\right)_{\mathrm{esc}} \Rightarrow \\
\Rightarrow 2\left[\mathrm{Cu}_{3} \mathrm{As}\right]_{\mathrm{eje}}+8[\mathrm{Cu}]_{\mathrm{eje}}+\left(\mathrm{Fe}_{3} \mathrm{O}_{4}\right)_{\mathrm{esc}}+3\left\{\mathrm{SO}_{2}\right\}_{\mathrm{gas}}
\end{gathered}
$$

Lo anterior podría explicar que, a medida que aumenta la cantidad de polvo recirculado, aumentan las cantidades de óxidos en la escoria, promoviendo la formación de arseniatos. De la misma forma, $\mathrm{Sb}$ puede reaccionar y formar antimonita de cobre $\left(\mathrm{Cu}_{2} \mathrm{Sb}\right)$, de acuerdo a:

$$
\begin{gathered}
6[\mathrm{FeS}]_{\mathrm{eje}}+\left(\mathrm{Sb}_{2} \mathrm{O}_{3}\right)_{\mathrm{esc}}+5\left[\mathrm{Cu}_{2} \mathrm{O}\right]_{\mathrm{esc}} \Rightarrow \\
\Rightarrow 2\left\{\mathrm{Cu}_{2} \mathrm{Sb}\right\}_{\mathrm{eje}}+6\{\mathrm{Cu}\}_{\mathrm{eje}}+2\left(\mathrm{Fe}_{3} \mathrm{O}_{4}\right)_{\mathrm{esc}}+3\left\{\mathrm{~S}_{2}\right\}_{\mathrm{gas}}
\end{gathered}
$$

Es posible observar que el equilibrio de las reacciones (4) y (5) depende fuertemente de las actividades del $\mathrm{As}_{2} \mathrm{O}_{3}$ y del $\mathrm{Cu}_{2} \mathrm{O}$ en la escoria. Analizando termodinámicamente la formación las reacciones anteriores, se pudo observar que la energía libre de Gibbs es muy negativa en un amplio rango de temperaturas, lo cual confirma que las reacciones entre los óxidos de arsénico y cobre con sulfuro de hierro se producen de manera casi espontánea, como se presenta esquemáticamente en la figura $10^{[12]}$. Otras impurezas presentes como $\mathrm{Zn}, \mathrm{Pb}$ y $\mathrm{Bi}$ pueden formar complejos con $\mathrm{Cu}$ y As, lo cual hace aumentar el nivel de impurezas en el eje.

Saarinen et al ${ }^{[13]}$ mostraron que durante el proceso de fusión, con rangos de metal desde 60 a 70 \% de $\mathrm{Cu}$, es posible que la formación de una fase metálica rica en impurezas precipite cuando existan altas concentraciones de metales como As, $\mathrm{Sb}, \mathrm{Bi}$ y $\mathrm{Pb}$ en el eje, denominado speiss. Este material tiene una densidad alta y un bajo punto de fusión (alrededor de $800{ }^{\circ} \mathrm{C}$ ), sedimentando en el fondo del reactor. Como posee una baja viscosidad puede penetrar las líneas de refractarios, generando problemas operacionales de importancia. Moyano et al. ${ }^{[14]}$ demostraron que la eliminación de As en la fase gaseosa tiende a estabilizarse a medida que aumenta la concentración de As en la carga (concentrado). Esta conducta se debe, principalmente, a la presencia de óxidos metálicos de $\mathrm{Fe}, \mathrm{Cu}, \mathrm{Zn}$ y $\mathrm{Pb}$, que en contacto con altas concentraciones de As tienden a formar arseniatos.

Nozawa ${ }^{[15]}$, en colaboración con los autores del presente trabajo, demostró que a temperaturas de entre $800-900 \mathrm{~K}$, los contenidos de $\mathrm{Cu}, \mathrm{Zn}, \mathrm{Pb}$ y Fe en

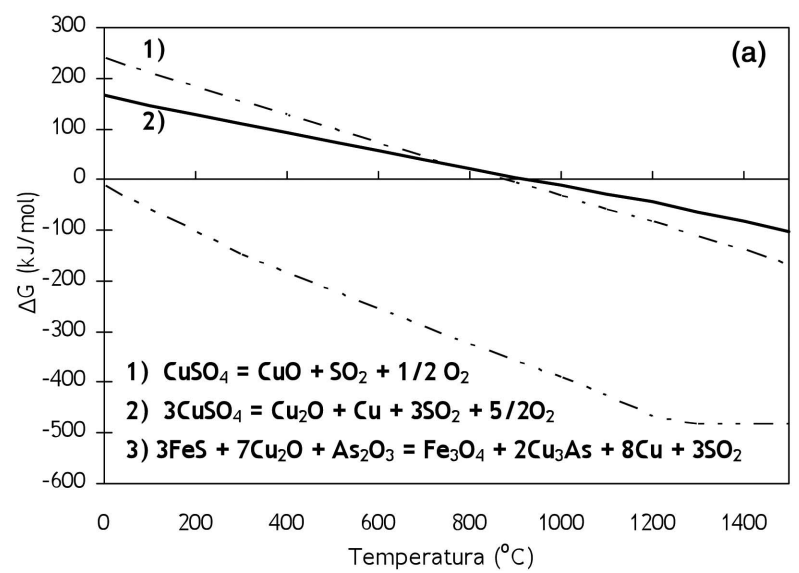

Figura 10. Energía libre de Gibbs para la formación de speiss como una función de la temperatura.(a) arsénico, y (b) antimonio.

Figure 10. Gibbs free energy for the formation of speiss as a function of the temperature. (a) arsenic, and (b) antimony.

contacto con As pueden formar arseniatos de cobre. Los resultados se presentan en la figura 11. A las temperaturas de operación y presiones parciales de oxigeno en la fusión de concentrado de cobre, tiende a formarse arseniato de cobre (speiss de cobre), el cual seria el causante de la disminución de la eliminación de impurezas, como se presenta en la figura 12 .

Lo anterior confirma que altas recirculaciones de polvos no son recomendables, ya que aumentan la concertación de las impurezas en el eje, especialmente

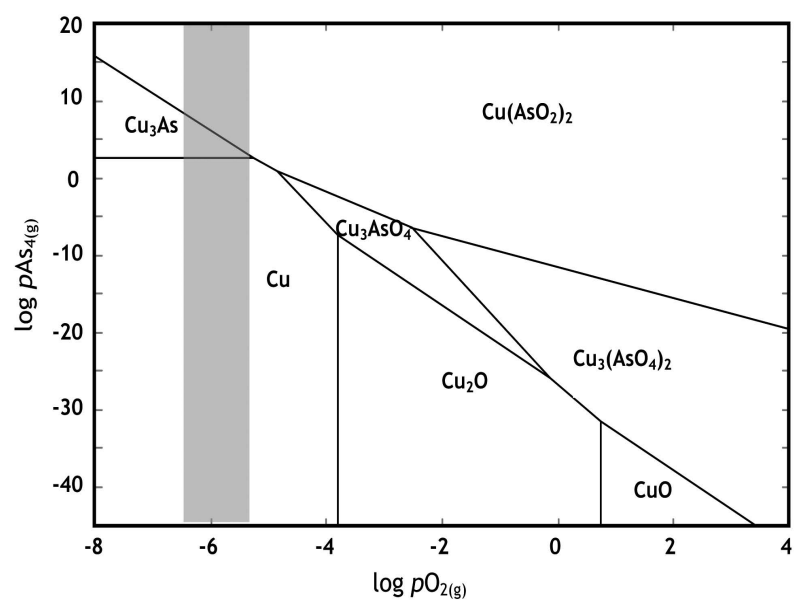

Figura 11. Diagrama de estabilidad de fases para Cu-As-O a $1.523 \mathrm{~K}^{[15]}$.

Figure 11. Phase stability diagram for $\mathrm{Cu}-\mathrm{As}-\mathrm{O}$ at $1,523 K^{[12]}$. 


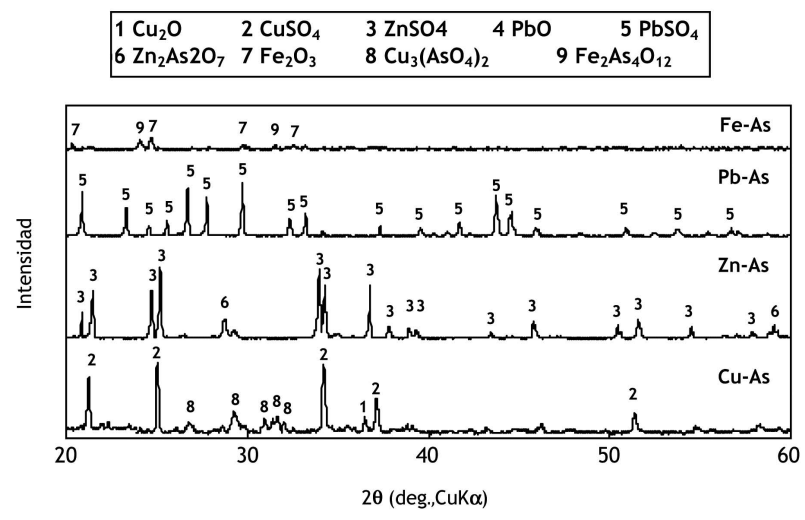

Figura 12. Resultados de difracción X-ray de formación de óxidos complejos de varios óxidos metálicos después de un proceso de tostación sulfhídrica a $800 \mathrm{~K}$.

Figure 12. X-ray diffraction patterns of complex oxide formation for various metal oxides after sulfating roasting process at $800 \mathrm{~K}$

para los casos de $\mathrm{Pb}$ y $\mathrm{Zn}$. Por lo tanto, la recirculación de polvos esta limitada a la calidad del eje.

\section{CONCLUSIONES}

El efecto de la recirculación de polvos al proceso de fusión en la generación de polvos y distribución de impurezas, entre las fases eje, escoria y polvo fue investigado a escala laboratorio bajo diferentes condiciones de proceso. La cantidad de polvo recirculado al proceso depende fuertemente de la calidad del eje a producir. Se encontró que la concentración en el eje de metales, tales como As, $\mathrm{Sb}$ y Bi, aumentó levemente con el polvo recirculado. Sin embargo, la concentración de $\mathrm{Pb}$ y $\mathrm{Zn}$ dependen fuertemente de la recirculación directa de polvo al proceso, limitando las recirculaciones al proceso.

Adicionalmente, la presencia de altas cantidades de As y Sb en los polvos puede provocar formación y precipitación de arseniatos y antimoniatos de cobre, los cuales pueden generar problemas operacionales de importancia.

\section{REFERENCIAS}

[1] A. Valenzuela, J. Palacios, D. Cordero y M. Sanchez, Proc. Yazawa Int. Symp., C. Yamauchi, and F. Kongoli (Eds.)TMS, The Minerals, Metals \& Materials Society, 2003, San Diego, CA, EE. UU., 2003, pp. 239-252.

[2] A. Yazawa, Proc. 28th Cong. IUPAC, Vancouver, Canadá, 1981, pp. 1-21.

[3] M. Maldini, J. Osorio, M. Mella y M. Herrera, Proc. Hydroprocess, Arturo Prat University, Iquique, Chile, 2006, pp. 402-414.

[4] S. Mikhail, A. Webster y J. Laflamme, Can. Metall. Q. 28 (1989) 241-249.

[5] T. Kho, D. Swinbourne y T. Lehner, Metall. Trans. B 37 (2006) 209-214.

[6] R. Alvarado, B. Lectora, F. Hernandez y C. Moya, Copper 95, Vol. 4, W.J. Chen, C. Diaz, A. Luraschi and P.J. Mackey (Eds.), Canadian Institute of Mining, Metallurgy and Petroleum, Quebec, Canadá, 1995, pp. 83-101.

[7] K. Itagaki, Metall. Rev. MMIJ 3 (1986) 87-100.

[8] A. Yazawa y T. Azakami, Can. Metall. Q. 8 (1970) 257-261.

[9] A. Yazawa, S. Nakazawa y Y. Takeda, Proc. Advances in Sulfide Smelting, H. Y. Sohn, D. B. George y A. D. Zunkel (Eds.), AIME, 1983, pp. 99-117.

[10] K. Itagaki y A. Yazawa, Proc. Advances in Sulfide Smelting, H. Y. Sohn, D. B. George y A. D. Zunkel (Eds.), AIME, 1983, pp. 119-142.

[11] K. Itagaki y A. Yazawa, Proc. Advances in Sulfide Smelting, H. Y. Sohn, D. B. George y A. D. Zunkel (Eds.), AIME, 1983, pp. 705-722.

[12] HSC Chemistry v.5, Outokumpu Research Oy., Pori, Finland.

[13] R. Saarinen y I. Kojo, US Patent 6858175 (2005).

[14] A. Moyano, C. Caballero, R. Mackay, K. Itagaki y J. Font, Proc. Sohn Int. Symp., Vol. 7. F. Kongoli and R. Reddy (Eds.), TMS, 2006, pp. 105-113.

[15] T. Nozawa, Tesis de Máster en Ciencias de la Ingeniería, Escuela de Ingeniería, Universidad de Nagoya, Japón, 2007. 